\title{
Aportes de LAS FUNDACIONES AFE AL DESARROLLO TERRITORIAL Y A LOS ODS
}

\author{
John Cubillos Nieto**
}

\section{Resumen}

El presente artículo aborda la responsabilidad social empresarial desde las 72 fundaciones de la Asociación de Fundaciones Empresariales (AFE) y el análisis de tres componentes: 1) las zonas de inversión social de las fundaciones AFE y los objetivos de desarrollo sostenible; 2) las áreas de trabajo de los proyectos de las fundaciones AFE y los tres pilares y seis objetivos del Plan Nacional de Desarrollo 2014-2018; 3) la convergencia de las áreas de trabajo de los proyectos de las fundaciones AFE con los objetivos de desarrollo sostenible. Las discusiones de este artículo son el resultado de la triangulación y el análisis de información de las fundaciones AFE, recolectada para los tres componentes mediante cuatro instrumentos: 1) una matriz de caracterización de sus 72 fundaciones; 2) una matriz de 790 proyectos ejecutados por ellas entre 2015-2018; 3) veinte entrevistas realizadas a directivos de las fundaciones AFE y stakeholders considerados relevantes para la investigación; 4) una encuesta online aplicada a las 72 fundaciones AFE con 40 respuestas.

Palabras clave: fundaciones; inversión social privada; desarrollo sostenible; oDs.

* Los insumos utilizados para este artículo provienen de la investigación intermaestrías en la modalidad Capstone realizada en el periodo 2018 y 2019 entre la Facultad de Finanzas, Gobierno y Relaciones Internacionales de la Universidad Externado de Colombia y la AFE, "Las fundaciones AFE en el marco del desarrollo sostenible en Colombia”, dirigida por el docente e investigador Óscar Iván Pérez H. y realizada por los estudiantes de la maestría en Responsabilidad Social y Sostenibilidad, María Alejandra Medina García; la maestría en Asuntos Internacionales, Brenda Lorena Escobar Cortés y Astrid Quintero Prieto; y la maestría en Gobierno y Políticas Públicas, Karen Lorena Amaya Ávila, John Cubillos Nieto, Eduardo Romero Sierra y Johana Polanco Lozano.

** Especialista en Gobierno, Gerencia y Asuntos Públicos. Coordinador Salud y Nutrición Latinoamérica, Venezuela y Crisis Regional, Fundación Acción contra el Hambre Espańa, Bogotá (Colombia). [johncubillosnieto@ gmail.com]; [https://orcid.org/0000-0002-9832-0170].

Recibido: 3 de julio de 2019 / Modificado: 29 de julio de 2019 / Aceptado: 20 de agosto de 2019

Para citar este artículo

Cubillos Nieto, J. (2020). Aportes de las fundaciones AFE al desarrollo territorial y a los ods. OPERA, 26, $149-171$. DOI: https://doi.org/10.18601/16578651.n26.08 


\section{CONTRIBUTION OF BUSINESS ASSOCIATION FOUNDATIONS TO TERRITORIAL DEVELOPMENT AND SDGS}

\section{Abstract}

This article addresses corporate social responsibility from the 72 business association foundations (AFE for their initials in Spanish) and the analysis of three components: 1 . The social investment areas of the AFE foundations and the Sustainable Development Goals (sDGs); 2. The Work Areas of the AFE foundations projects and the 3 pillars and the 6 objectives of the National Development Plan 2014-2018; 3. The Convergence of the Work Areas of the AFE Foundations projects with the sDGs. The discussions in this article are the result of the triangulation and information analysis of the AFE foundations, collected for the 3 components by means of 4 instruments: 1. A characterization matrix for 72 foundations; 2 . A matrix of 790 projects executed by them, between 2015 and 2018; 3. 20 interviews with executives of the AFE foundations and stakeholders considered relevant to the research; and 4. An online survey applied to the 72 AFE Foundations with 40 responses.

Key words: Foundations; private social investment; sustainable development; sDGs.

\section{INTRODUCCIÓN}

Según el Plan Nacional de Desarrollo 2018-2022, "Pacto por Colombia, pacto por la equidad", el país presenta un cambio social notable en este siglo, con una fuerte caída de la pobreza de 49,7\% en 2002 a 27\% en 2017. Sin embargo, persisten un conjunto de factores y de restricciones que de no enfrentarse exitosamente comprometen la continuidad del cambio social y la posibilidad de lograr un país con mayor equidad y calidad de vida para todos (DNP, 2019). En Colombia, la incidencia de la pobreza monetaria en 2017 se situó en $26,9 \%$, con lo que se estima que 12,8 millones de personas en el país la padecen; adicional a esto, 3,5 millones de personas se encuentran en situación de pobreza extrema, principalmente indígenas, afros, personas en situación de desplazamiento y población rural (PNUD, 2018).

Uno de los actores más representativos de la inversión social privada en Colombia es la Asociación de Fundaciones Familiares y Empresariales (AFE), gremio de las fundaciones familiares y empresariales, cuya misión es contribuir al fortalecimiento de sus asociadas mediante el desarrollo de capacidades, el fomento del trabajo colaborativo y la incidencia en prácticas del sector privado y en políticas públicas.

Este artículo pretende responder la pregunta ¿cómo se articulan las fundaciones que hacen parte de la AFE con el desarrollo en los territorios? Para resolverlo se analiza la manera como las fundaciones pertenecientes a la AFE se articulan en los territorios desde sus proyectos de inversión, con el PND 2014-2018 y los 17 objetivos de desarrollo sostenible (ODS) - CONPES 3918 (DNP, 2018).

Como hipótesis se plantea que las fundaciones AFE no llevan una coherencia entre la focalización de sus proyectos y su alineación temática con las prioridades del Estado y los oDs, por lo cual un direccionamiento estratégi- 
co de su inversión social dentro de un enfoque integral de sostenibilidad desde lo económico y financiero, lo ambiental, lo social y lo institucional (BID, 2018), estaría más orientado a las necesidades reales que marcan las brechas en los territorios. La caracterización de lo que están haciendo las fundaciones AFE en los territorios permite visibilizar la construcción articulada de una ruta sinérgica hacia el desarrollo sostenible.

Las discusiones de este artículo se presentan desde los tres componentes: 1) las zonas de influencia de las fundaciones AFE y las zonas de inversión social; 2) las áreas de trabajo de los proyectos de las fundaciones AFE y el PND 2014-2018; 3) la convergencia de las áreas de trabajo de los proyectos de las fundaciones AFE con los ods, desde un enfoque integral de sostenibilidad. Para concluir, el artículo muestra los aportes que hace al conocimiento sobre el sector fundacional.

\section{DESARROLLO METODOLÓGICO}

Para resolver el interrogante de investigación, la ruta metodológica inicia con la revisión bibliográfica de los conceptos de fundación, los tipos de fundaciones, las definiciones de enfoque territorial y de desarrollo sostenible. Progresivamente, se incorpora la georreferenciación de las zonas de influencia de las fundaciones AFE resultado de la clasificación de los proyectos de estas fundaciones por departamentos, y se continúa con el porcentaje de cambio requerido para el logro de los ods por departamentos en 2030, con base en los cálculos del Departamento Nacional de Planeación (DNP) a partir de las metas del CONPES 3918. Seguidamente, se trata el porcentaje de participación del producto interno bruto (PIB) de cada departamento reportado por el Departamento Administrativo Nacional de Estadística (DANE) entre 2014 y 2017, para luego hablar de los resultados del cruce entre las áreas de trabajo de los proyectos de las fundaciones AFE identificadas en su matriz de proyectos, los pilares y objetivos del Plan Nacional de Desarrollo (PND) 2014-2018 y los 17 oDs hilando el tema del desarrollo sostenible en los tres momentos.

Las fuentes de información tomadas en cuenta para este artículo corresponden a las de una investigación mixta que comprende datos cualitativos suministrados por la AFE, consolidados en una matriz de caracterización de las 72 fundaciones AFE, y una matriz de 790 proyectos ejecutados entre 2015 y 2018 , actualizados con nuevas variables relevantes para la investigación y adicionando nueva información reportada por las fundaciones en sus informes de gestión, informes de sostenibilidad y en sus páginas web; solo se incluyeron en el estudio los proyectos con información en la mayoría de las variables establecidas. También incluye los resultados de 20 entrevistas realizadas a directivos de las fundaciones AFE (fundaciones como Corona, Cerrejón, Alpina, Éxito, Carvajal, Haceb, Surtigas, Uniban, Pintuco, Antonio Restrepo Barco, Pro Antioquia, Promigas, Carulla, entre otras) y stakeholders considerados relevantes para la investigación, entre ellos funcionarios que trabajan temas de cooperación o alianzas en instituciones como la Agencia Presidencial de Cooperación (APC-Colombia), el Instituto Colombiano de 
Bienestar Familiar (ICBF), o el Banco Interamericano de Desarrollo (BID). Por último, el trabajo incluye datos cuantitativos provenientes de una encuesta online aplicada a las 72 fundaciones AFE con 40 respuestas que fueron tabuladas por temas específicos para explorar sobre sus intencionalidades, sus actividades y su relacionamiento con el Estado, los modelos de inversión social que manejan y su alineación frente a los oDs.

El análisis que se presenta en las siguientes páginas es el resultado de la triangulación de los hallazgos provenientes de las diferentes fuentes de información mencionadas, bajo el manto del desarrollo sostenible con un enfoque territorial.

\section{APROXIMACIONES CONCEPTUALES}

Inicialmente, se describen las fundaciones como entidades independientes no estatales que emplean sus recursos privados asociándose y canalizándolos dentro del financiamiento o la ejecución de programas propios a favor del progreso social, cultural, económico, ambiental, científico y otros propósitos caritativos de carácter público a nivel local, regional o internacional (OCDE, 2016). Las fundaciones familiares son entidades privadas independientes, establecidas por una familia con fondos que provienen de ella, en las que los familiares hicieron o hacen contribuciones al patrimonio y también pueden ser miembros de su máximo órgano de gobierno (Villar, 2018, p. 18). La fundación empresarial es una persona jurídica, creada por una organización empresarial, con patrimonio propio, sin ánimo de lucro, orien- tada hacia la atención de necesidades públicas y el bien común, y que, aunque tiene afiliación con la empresa es independiente de ella (Fundación Promigas y Fundación DIs, 2012).

Para abordar los datos y las discusiones presentadas en este artículo, es necesario hacer algunas precisiones conceptuales alrededor de los planteamientos de autores u organizaciones, que pueden contribuir a la comprensión del lector en el desarrollo de estos temas. En este sentido, es necesario precisar la definición de fundaciones, enfoque territorial e inversión social privada, para abordar los modelos de inversión de las fundaciones AFE, el ciclo de políticas públicas y la incidencia de las fundaciones y su relacionamiento con el Estado y, finalmente, el desarrollo sostenible y los ods para poder hablar de las fundaciones AFE y su relación con estos.

En el concepto de territorio se incorpora la apropiación del espacio por la acción social de diferentes actores, y se agrega el "juego de poder" entre los actores que actúan en un espacio. Como resultado del "juego de poder" se define una identidad relacionada con los límites geográficos o con un espacio determinado. El territorio surge, por tanto, como resultado de una acción social que, de forma concreta y abstracta, se apropia de un espacio física y simbólicamente (Flores, 2007, p. 36).

Seguidamente, para este escrito se acoge la definición de la Organización de las Naciones Unidas para la Alimentación y la Agricultura (FAO) que concibe el enfoque territorial como un proceso de planificación aplicado a un territorio socialmente construido, de amplia interrelación y consenso entre actores institucionales y sociales, que se identifican con un 
espacio geográfico, donde viven y operan, por tradición cultural, política e histórica, para resolver problemas comunes y solucionarlos de acuerdo con intereses y prioridades compartidas. (FAO, 2013). Esta definición recoge los conceptos anteriores de una forma sintética aplicable a este ejercicio.

Sobre el tema de inversión social privada es necesario hablar un poco del concepto desde las fundaciones. Las fundaciones AFE invierten en proyectos sociales de diferentes formas, con recursos financieros, capital humano, experiencia específica o diferentes aportes en especie (Villar, 2015). Esta inversión, por sus características y fines, se define como inversión social privada (ISP), entendida como una asignación estratégica de recursos privados para beneficio público; esta inversión describe la entrega de recursos financieros y no financieros de la organización a la comunidad o a causas sociales (Chávez, Yepes y Cannon, 2012, p. 19).) Este tipo de inversión "implica la destinación de recursos privados a mejorar las condiciones de vida de comunidades y/o grupos de población específicos, a partir de una decisión voluntaria por razones filantrópicas o de responsabilidad social" (Carvajalino, 2007, p. 3). Este autor también destacó que se "habla de una inversión, no de un gasto" (p. 3).

Por su parte, las inversiones de impacto son realizadas por cualquier tipo de organización, compañía, fondo o fundación, con la intención de generar un impacto social y ambiental positivo, junto con un rendimiento financiero (OECD, 2015), que puede estar por debajo de la tasa de mercado según los objetivos estratégicos de los inversores (GIIN, 2017). Aproximadamente, en los últimos cinco años las fundaciones AFE usan este tipo de inversión orientadas por una visión más estratégica que contempla no solo el riesgo y el retorno, sino también el impacto. Así logra generar innovaciones positivas que produzcan un cambio real en la calidad de vida de las personas (Inversión de Impacto, 2017).

Para entender el relacionamiento de las fundaciones AFE con el Estado es importante ubicarse en el modelo secuencial de las políticas, el cual contempla varias etapas que van desde la identificación de un problema público a la inclusión de este en la agenda pública, la identificación, negociación, selección y diseño de la mejor alternativa, para luego implementarla con un sistema de monitoreo y evaluación que permita su ajuste, reformulación o terminación dentro de un proceso de retroalimentación itinerante (Ordóńez, 2013).

En el proceso de una política pública resulta valiosa la participación de un actor de las organizaciones civiles diferente al gubernamental, esto genera valor agregado ya que estas organizaciones son las que muestran las necesidades y demandas que deben ser tenidas en cuenta en la política pública. Para ello, es necesario un proceso premeditado y sistemático que consiste en planear y realizar un conjunto de acciones orientadas a influir en la toma de decisiones políticas, proceso conocido como incidencia en políticas públicas (Sinigaglia et al., 2006).

En lo referente a la alineación temática de las fundaciones con las prioridades del Estado es importante precisar que la alineación o no de los temas tratados por los proyectos de las fundaciones AFE y las prioridades del Estado puede favorecer de alguna manera los procesos 
de control político o de rendición de cuentas del Gobierno. La rendición de cuentas (accountability) implica la obligación de informar detalladamente y asumir responsabilidades sobre una determinada acción o conjunto de acciones. Involucra tres dimensiones: 1 ) informativa, que se refiere a dar a conocer determinadas acciones; 2) explicativa, que concierne a dar razones de lo que se ha llevado a cabo; 3) de evaluación, en el sentido de reconocer lo correcto y cuestionar lo erróneo (Isunza, 2006). El Banco Mundial ha reconocido el accountability como "una perspectiva para construir rendición que se sustenta en el involucramiento cívico, en el que los ciudadanos comunes y/o las organizaciones civiles son los que participan directa o indirectamente en la rendición” (2004, citado en Natal, 2006, p. 7).

Para introducir el tema del desarrollo sostenible es vital enfocarse en lo que hacen las fundaciones desde sus intencionalidades. Todas las fundaciones AFE cuentan con fines sociales definidos desde la inversión social o el desarrollo de proyectos que buscan mejorar problemáticas que le impiden a las personas desenvolverse o vivir de una manera saludable que amplíe sus posibilidades frente a aquellos factores que puedan interferir con su bienestar y su calidad de vida. Dentro de los avances que hemos logrado como sociedad está la inclusión del desarrollo sostenible en cualquier acción que se lleve a cabo; independientemente de su fin, cualquier actividad genera afectaciones positivas o negativas al medio ambiente a corto y largo plazo, de ahí la relevancia de incluir este tema al tratar el quehacer de las fundaciones AFE (Haro-Martínez y Taddei-Bringas, 2014).
El desarrollo sostenible es un concepto definido originalmente en el Informe Brundtland de 1987, elaborado por distintas naciones, y que se refiere al desarrollo que satisface las necesidades de la generación presente, sin comprometer la capacidad de las generaciones futuras de satisfacer sus propias necesidades. La redacción de esta premisa supuso un cambio social, ambiental y económico importante que, además, aludía a discusiones morales sobre el medioambiente que nunca antes habían sido debatidas (ONU, 2017).

Con el fin de implementar medidas y objetivos para tener un mundo mejor, se plantearon los 17 ods, los cuales tienen como fin el crecimiento de las naciones, acabar con la pobreza, proteger el planeta y garantizar la prosperidad para todos dentro de una visión integral de desarrollo. Los oDs se gestaron en la Conferencia de las Naciones Unidas sobre el Desarrollo Sostenible celebrada en Río de Janeiro en 2012. El propósito de la Conferencia era crear una agrupación de objetivos mundiales con relación a los desafíos ambientales, políticos y económicos a los que se enfrenta el mundo (PNUD, 2019). En septiembre de 2015, los 193 países pertenecientes a la ONU adoptaron objetivos con el propósito de: 1) erradicar la pobreza, 2) proteger el planeta y 3) garantizar la prosperidad para todos. Cada uno de los 17 objetivos tiene metas específicas, las cuales deben ser logradas en 2030 (DNP, 2017).

Dada la complejidad de los problemas actuales, para el logro de los 17 ods en 2030 se requiere de un manejo integral como el que plantea el BID en su informe de sostenibilidad 2018, que abarca temas económicos y financieros, ambientales, sociales e institucionales. 
En los temas económicos y financieros orienta sobre la asignación justa y transparente de los riesgos, sobre el apoyo al crecimiento incluyente y sostenible, el impulso a la productividad, y la recuperación adecuada de los costos (retornos en impacto social o en especie). En los temas ambientales orienta sobre el uso sostenible y eficiente de los recursos naturales (sistemas de ahorro de luz y agua, reutilizar, reciclar y reducir), el poner límites a la contaminación y sobre la resiliencia a los riesgos climáticos y desastres naturales. En los temas sociales orienta sobre el cumplimiento de los derechos humanos y laborales, la salud, la seguridad, la igualdad de género y la diversidad, la contribución para mejorar las vidas, el bienestar social, la inclusión, la equidad y la transparencia. Por último, para los temas institucionales trata sobre la alineación con los compromisos nacionales e internacionales, el desarrollo de la capacidad local, los sistemas de recopilación de datos, el monitoreo y la evaluación, y el fortalecimiento institucional y la generación de conocimiento (BID, 2018).

\section{RESULTADOS}

A continuación, se presentan los resultados de la información obtenida a partir de las diferentes fuentes utilizadas para este artículo organizada por los tres componentes de la investigación: 1) las zonas de inversión social de las fundaciones AFE y los oDs; 2) las áreas de trabajo de los proyectos de las fundaciones AFE y los tres pilares y los seis objetivos del PND 2014-2018; 3) la convergencia de las áreas de trabajo de los proyectos de las fundaciones AFE con los oDs.

\section{Zonas de inversión de las fundaciones AFE y los ODS}

Partiendo de la matriz de los 790 proyectos de las fundaciones AFE realizados entre 2015 y 2018, estos se clasificaron por departamentos y se georreferenciaron (figura 1).

En esta georreferenciación se evidenció que el $52 \%$ de los 790 proyectos que realizan las fundaciones AFE se concentra en la zona central del país, principalmente en tres departamentos: Valle del Cauca (21\%), Antioquia (20\%) y Atlántico (11\%). Al revisar por tipo de fundación, el $71 \%$ de los proyectos son de fundaciones empresariales, $23 \%$ familiares y $6 \%$ independientes.

$\mathrm{Al}$ relacionar la matriz de proyectos de las fundaciones AFE en cuanto a los departamentos donde se ejecutan y la localización de las sedes de las empresas que tienen fundaciones empresariales, se identificó que el $81 \%$ de los proyectos se desarrollan en departamentos de zonas de influencia. Un ejemplo es la fundación Caicedo González Riopaila Castilla que concentra su inversión en el Valle del Cauca, departamento en donde se ubica la sede principal del grupo agroindustrial Riopaila Castilla, que produce y comercializa azúcar, miel, alcohol, energía y palma.

Si bien la tendencia de localización de la inversión social es clara, en entrevistas con algunos directores de las fundaciones, estos manifestaron hacer también inversión en territorios diferentes a las zonas de influencia a 


\section{FIGURA 1. MAPA DE DISTRIBUCIÓN DE PROYECTOS DE LAS FUNDACIONES AFE POR DEPARTAMENTOS}

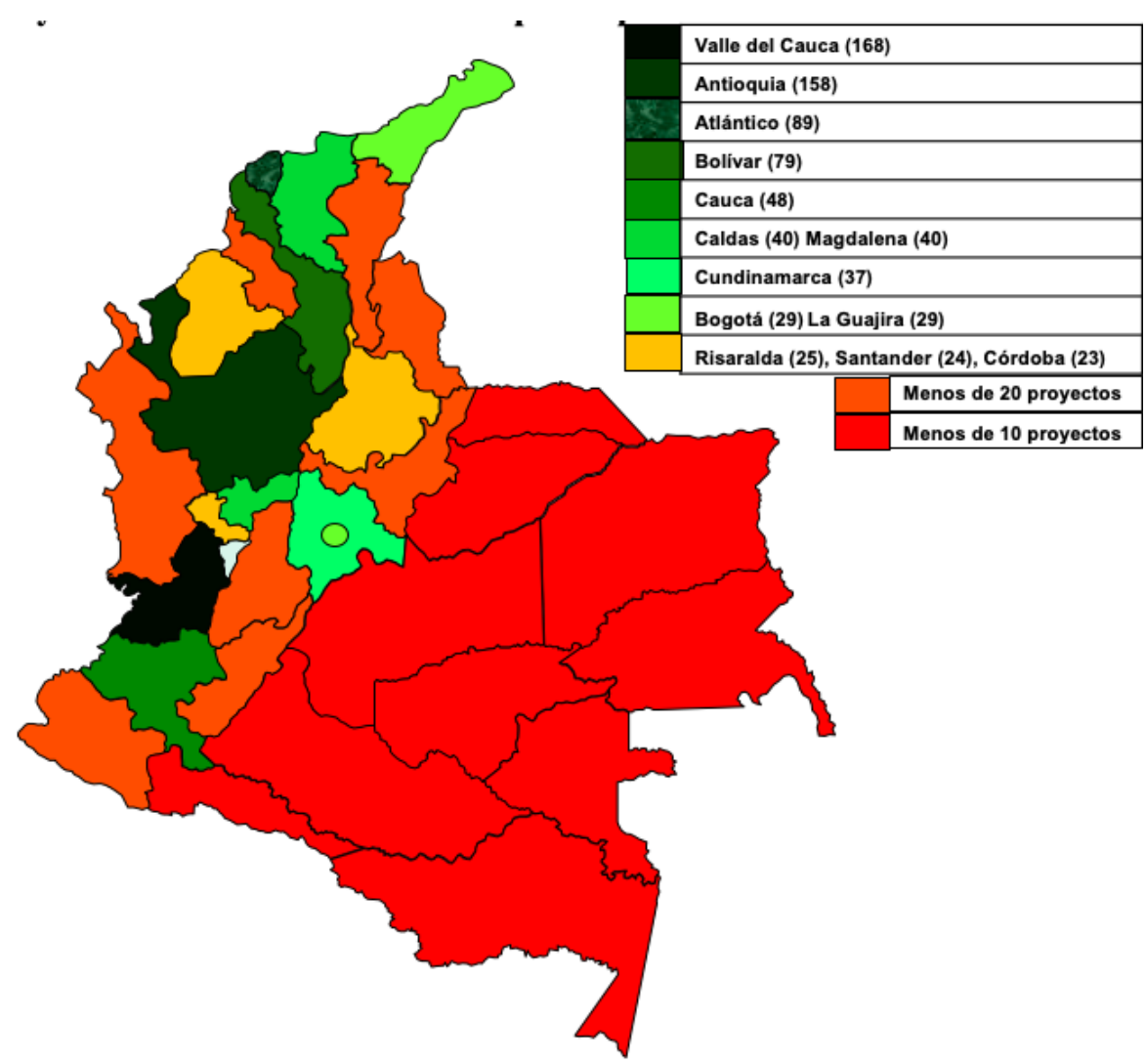

Fuente: elaboración equipo Capstone, Universidad Externado de Colombia-AFE, 2019, con base en información suministrada por la AFE en desarrollo de la consultoría.

través de alianzas con otras fundaciones o instituciones. Sin embargo, el hecho de responder al interés de una empresa limita el trabajo de las fundaciones, así lo manifestó un director de una fundación familiar al ser entrevistado:

La empresa tiene un área de responsabilidad social empresarial, pero la fundación es totalmente familiar e independiente, lo que te permite a ti es no estar en zonas de influencia, no depender de las empresas, si no, depender de lineamientos a largo plazo, tener más autonomía, más capacidades de arriesgarte. (Director ejecutivo fundación familiar, comunicación personal, marzo de 2019)

Por otro lado, en departamentos rezagados frente al cumplimiento de indicadores y metas sociales como Chocó, Sucre, Norte de Santander, Boyacá, Tolima, Huila y Quindío, las fundaciones AFE hacen presencia con menos de 20 proyectos. Peor aún sucede en el sur y el oriente del país en departamentos como 
Meta, Casanare, Arauca, Vichada, Guainía, Guaviare, Caquetá, Vaupés, Putumayo y Amazonas, en donde la presencia de los proyectos es más baja, pues cuentan con menos de diez proyectos por departamento, por las razones expuestas más adelante en las entrevistas.

Para ahondar en la pertinencia territorial de la focalización de los proyectos de las fundaciones AFE, se indagó sobre el porcentaje de participación del PIB por departamentos entre 2014 y 2017 para cruzarlo con el número de proyectos ejecutados por las fundaciones en los mismos. El análisis evidenció que aquellos departamentos en los que los proyectos de las fundaciones AFE tienen mayor presencia, también cuentan con mayores porcentajes de participación en el PIB nacional, por ende, cuentan con un mayor grado de desarrollo productivo. Ejemplos de esto son Antioquia (PIB 14,5; 2017) y Valle del Cauca (PIB 9,8;
2017), que cuentan con más de 150 proyectos de las fundaciones AFE. En contraste, en donde se observa menor porcentaje del PIB y, por tanto, menor productividad y menores recursos para atender problemas sociales, hay menor presencia de los proyectos de las fundaciones AFE (figura 2).

Cuando se exploró en las entrevistas por las razones que causaban esta situación, en diálogo con un asesor del ICBF - uno de los stakeholders identificados en el Gobierno nacional-, refirió que

... los sobrecostos para ejecutar proyectos en las zonas rurales, rural dispersas y en algunas zonas urbanas, no permitían hacer un buen uso de los recursos, puesto que los gastos administrativos, de transporte, de intermediarios, de traslado de talento humano calificado y demás requisitos para operar copan la mayor parte de los recursos limitando el impacto de las intervenciones,

\section{FIGURA 2. PORCENTAJE PROMEDIO DEL PIB POR DEPARTAMENTO 2014-2017 FRENTE A PORCENTAJE DE PROYECTOS DE LAS FUNDACIONES AFE POR DEPARTAMENTOS}

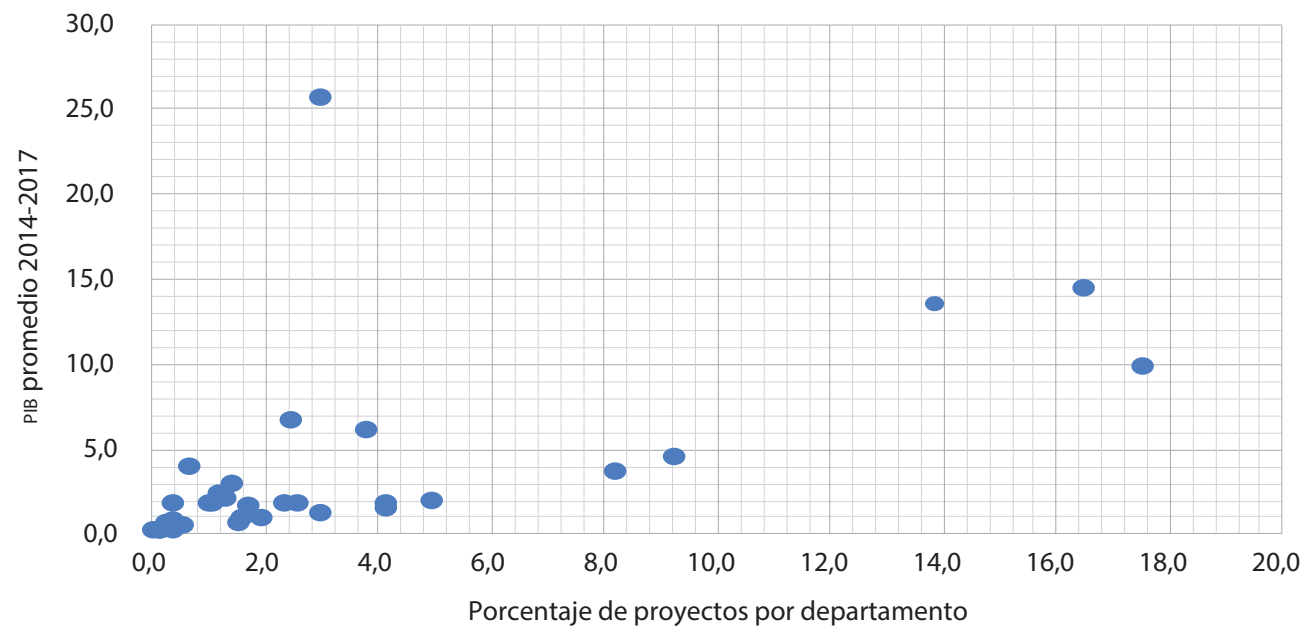

Fuente: elaboración equipo Capstone, Universidad Externado de Colombia-AFE, 2019, con base en información suministrada por la AFE. 
por lo cual casi todo el presupuesto se consume en la operación y no llega a los beneficiarios. (Asesor de la Oficina de Cooperación y Convenios, comunicación personal, 20 de marzo de 2019)

Las entrevistas también aportaron sobre la concentración de proyectos por departamentos presentados en la figura 1 ; frente a esto, las fundaciones y los grupos de interés relacionaron algunos criterios para elegir los territorios en los que operan o apoyan con recursos. Dentro de estos criterios dos de los entrevistados mencionaron: que los territorios cuenten con otros aliados estratégicos, que tengan posibilidades de apalancamiento económico, que tengan presencia del Estado para garantizar la seguridad de la operación, que cuenten con voluntad política para cooperar y que los gastos administrativos no sobrepasen la inversión final destinada a los beneficiarios (director fundación familiar, comunicación personal, 01 de marzo de 2019 y asesor de la Oficina de Cooperación y Convenios, comunicación personal, 20 de marzo de 2019). En contraste, al cruzar la ubicación de las fundaciones y sus zonas de influencia con los departamentos con los PIB más altos y la concentración de proyectos, todos estos se superponen. Así las cosas, los departamentos más rezagados, que no cumplen estos criterios, se encuentran limitados para recibir más apoyos por parte de las fundaciones y el Estado.

Con el objetivo de relacionar la focalización de la inversión social que hacen las fundaciones AFE en los departamentos con el cumplimiento de los oDs, se incorporó en la discusión el CONPES 3918 de 2018, el cual define 16 metas a 2030, y los cálculos que realizó el DNP para establecer en porcentaje el cambio requerido por departamento para lograr estas metas a 2030. En la tabla 1 se relacionan estas cifras con los proyectos por departamentos.

Como se observa en la tabla 1 , los departamentos de Atlántico, Bolívar, Cesar, Sucre, Córdoba, La Guajira y Magdalena presentan mayores brechas para el logro de los ods 1, 2, 3, 4, 5, 6, 9, 10 en 2030, aquí las fundaciones AFE aparecen con menos de 100 proyectos; para los departamentos de Chocó, Nariño y Cauca el reto está en los oDs 1, 3, 5, 9,10,11; para los departamentos de Meta, Casanare, Arauca, Vichada, Guainía, Guaviare, Caquetá, Vaupés, Putumayo y Amazonas, donde las fundaciones AFE hacen presencia con menos de 10 proyectos, el reto está en los ods 3, 4, 7, 16. Con estos resultados se evidencia la incongruencia entre el número de proyectos de las fundaciones AFE por departamentos y las necesidades que marcan sus brechas -con la excepción de Valle del Cauca, donde las fundaciones AFE implementan 168 proyectos, focalización pertinente para que el departamento supere sus limitaciones en el logro de los ods 3,5,10 y 11 -. En este punto se considera deseable alinear las áreas de trabajo de las fundaciones AFE con los temas de los oDs rezagados en cada uno de los departamentos.

\section{Áreas de trabajo de los proyectos de las fundaciones AFE y su alineación con el Plan Nacional de Desarrollo 2014-2018 y los oDs}

Un factor determinante para reconocer el aporte al desarrollo territorial que hacen las fundaciones AFE frente a objetivos y metas nacionales es la correlación que existe entre la alineación temática de proyectos de las fundaciones AFE con los temas priorizados por 
TABLA 1. FOCALIZACIÓN DE LAS ACCIONES DE LAS FUNDACIONES AFE Y EL CAMBIO PARA LOGRAR LA META DE LOS ODS A 2030 EN PUNTOS PORCENTUALES SEGÚN EL DNP

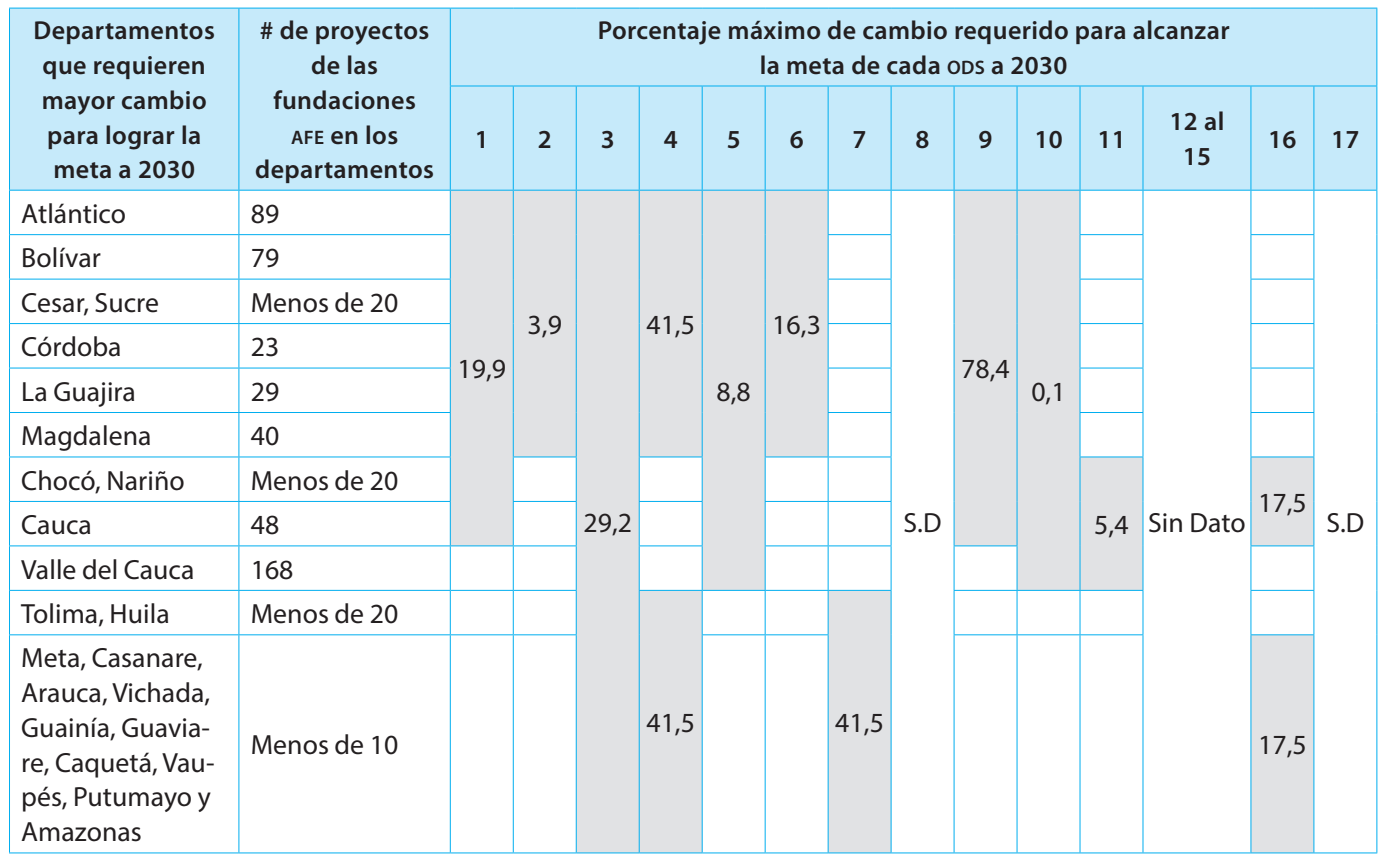

Fuente: elaboración propia a partir de los resultados de la matriz de proyectos de las fundaciones AFE y los cálculos reportados por el DNP para alcanzar las metas trazadoras de cada uno de los oDs en 2030 (DNP, 2019).

el Estado. Una alineación temática implica la armonización de propósitos entre el sector fundacional y el Estado, lo que favorece la articulación y la gestión para el desarrollo.

Para buscar esta correlación se cruzaron las áreas de trabajo de los proyectos de las fundaciones AFE con los temas que desarrollan los tres pilares y los seis objetivos del PND 20142018, y luego se analizaron los resultados desde la triangulación de cuatro fuentes de información: 1) los resultados de la tabulación de una encuesta online aplicada a las 72 fundaciones AFE, en donde el $56 \%$ (40 fundaciones) diligenció el cuestionario y envió sus respuestas; 2) los resultados de la matriz de 790 proyectos de las fundaciones AFE filtrando sus áreas de trabajo;3) 20 entrevistas realizadas a directivos de las fundaciones AFE y stakeholders considerados relevantes para la investigación, y 4) el documento técnico del PND 2014-2018. A continuación, se presentan y desarrollan los resultados de esta convergencia.

El pND 2014-2018, “Todos por un nuevo país", del gobierno del presidente Juan Manuel Santos, en la visión apuntaba hacia "una sociedad en paz que pueda focalizar sus esfuerzos en el cierre de brechas y que pueda invertir recursos en mejorar la cobertura y calidad de su sistema educativo, envuelto en una estrategia de crecimiento verde". Este Plan trabajó tres pi- 
lares: paz, equidad y educación; y seis objetivos: 1) competitividad e infraestructura estratégicas; 2) movilidad social; 3) transformación en el campo; 4) seguridad, justicia y democracia; 5) buen gobierno y 6) crecimiento verde (PND 2014- 2018). A continuación, en la tabla 2 se relacionan los tres pilares el PND 2014-2018 con la clasificación de las áreas de trabajo de la base de proyectos de las fundaciones AFE desde un análisis de correlación entre el texto y los planteamientos temáticos.

De acuerdo con estos resultados, hay una alineación de cuatro áreas de los proyectos de las fundaciones AFE con el pilar 1: Colombia en paz, que se orienta a temas como la construcción de paz, la integración territorial y comunitaria para cerrar brechas poblacionales y sociales, el potenciamiento de la conectividad para la inclusión productiva, el acceso a bienes y servicios, y la reducción de desigualdades sociales mediante el desarrollo integral del campo. Igualmente, hay 21 áreas que se alinean con el pilar 2: Colombia equitativa y sin pobreza extrema, este pilar busca erradicar la pobreza extrema a 2024 y reducir la moderada, al igual que reducir brechas en materia de ingresos, servicios de calidad en salud, educación, servicios públicos, infraestructura

\section{TABLA 2. CONVERGENCIA DE LAS ÁREAS DE TRABAJO DE LOS PROYECTOS DE LAS FUNDACIONES AFE CON LOS TEMAS DE LOS PILARES DEL PLAN NACIONAL DE DESARROLLO 2014-2018}

\begin{tabular}{|c|c|c|c|}
\hline & Pilar 1 & Pilar 2 & Pilar 3 \\
\hline Áreas de trabajo de los proyectos de las fundaciones AFE & $\begin{array}{l}\text { Colombia } \\
\text { en paz }\end{array}$ & $\begin{array}{l}\text { Colombia equitativa } \\
\text { y sin pobreza } \\
\text { extrema }\end{array}$ & $\begin{array}{l}\text { Colombia } \\
\text { la más } \\
\text { educada }\end{array}$ \\
\hline $\begin{array}{l}\text { Personas y paz: Cultura y convivencia ciudadana, Democracia y partici- } \\
\text { pación ciudadana, Acceso a la justicia y Resolución pacífica de conflictos, } \\
\text { Paz, superación del conflicto y DD.HH. }\end{array}$ & 4 & & \\
\hline $\begin{array}{l}\text { Personas: Educación preescolar, educación básica y media, educación su- } \\
\text { perior y becas, educación técnica, tecnológica y Formación para el trabajo }\end{array}$ & & & 6 \\
\hline $\begin{array}{l}\text { Paz: Fortalecimiento y entorno institucional } \\
\text { Prosperidad: Espacios públicos e infraestructura comunitaria, Ciencia, } \\
\text { investigación y tecnología, Generación de ingresos y desarrollo pro- } \\
\text { ductivo, Emprendimiento, Recreación, deporte y uso del tiempo libre, } \\
\text { Arte y cultura } \\
\text { Asociaciones: Incidencia en políticas públicas, Promoción de volun- } \\
\text { tariado } \\
\text { Personas: Desarrollo de base y trabajo con comunidades, Seguridad } \\
\text { alimentaria y nutrición, Salud y protección social, Vida en familia y tejido } \\
\text { social,Vivienda y habitabilidad, Inclusión y educación financiera, Gestión } \\
\text { del conocimiento, Agua y saneamiento básico } \\
\text { Planeta: Donaciones y dotación, Gestión ambiental y medio ambiente, } \\
\text { Conservación y desarrollo sostenible }\end{array}$ & & 21 & \\
\hline $\begin{array}{l}\text { Número total de áreas de trabajo que convergen con cada objetivo } \\
\text { del PND }\end{array}$ & 4 & 21 & 6 \\
\hline
\end{tabular}

Fuente: elaboración equipo Capstone Universidad Externado de Colombia-AFE 2019, con base en los resultados de la base de proyectos y el PND $2014-2018$. 
y conectividad. También busca promover el desarrollo económico incluyente del país y sus regiones. Finalmente, el pilar 3: Colombia la más educada, busca cerrar las brechas en acceso y calidad a la educación acercando al país a altos estándares internacionales, igualando oportunidades para individuos, comunidades y regiones. Se evidenció que hay seis áreas que se alinean con este pilar.

Con estos resultados se puede concluir que las áreas de trabajo de los proyectos de las fundaciones AFE se alinearon con el PND 2014-2018, en la medida en que guardan relación directa o explícita con los tres pilares del Plan asociados a temas de paz, equidad y educación, así como también lo evidenciaron los resultados de la encuesta $(55,3 \%$ refieren estar alineados) y los de las entrevistas, donde más del $60 \%$ de entrevistados afirmaron estar alineados o haber tenido presentes los temas del plan de desarrollo para orientar sus acciones o proyectos.

El PND 2014-2018 también cuenta con seis objetivos nacionales que se cruzaron con la clasificación de las áreas de trabajo de la base de proyectos de las fundaciones AFE desde un análisis de relacionamiento textual y cualitativo. En la tabla 3 se presentan los resultados.

En la tabla 3 se puede ver claramente que seis áreas de los proyectos relacionadas con temas de infraestructura, fortalecimiento institucional, ciencia, tecnología y formación para el trabajo se alinean con el Objetivo 1: Competitividad e infraestructura estratégicas. Este objetivo apunta a incrementar la productividad de las empresas mediante el desarrollo productivo, la ciencia, la tecnología y la innovación para mejorar la competitividad; también busca promover la infraestructura vial, de transporte y servicios de logística. El desarrollo mineroenergético y el acceso a energías sostenibles son otras de las preocupaciones de este objetivo.

En cuanto al Objetivo 2: Movilidad social, se identificaron 23 áreas alineadas que trabajan temas de necesidades sociales que convergen. Dicho objetivo apunta a garantizar los mínimos vitales y avanzar en el fortalecimiento de las capacidades de la población en pobreza extrema para su efectiva inclusión social y productiva (Sistema de Promoción Social). Asimismo, mejorar las condiciones de salud, empleo, educación, vivienda, agua potable y saneamiento básico, bajo el concepto de "ciudades amables y sostenibles para la equidad", en complemento con las acciones estratégicas de movilidad urbana.

Entre tanto, en el Objetivo 3: Transformación en el campo, hay dos áreas que se alinean con temas de seguridad alimentaria y nutrición, y emprendimiento. Este objetivo busca ordenar el territorio rural mejorando el acceso a la tierra para los productores agropecuarios, cerrar brechas urbano-rurales, impulsar la competitividad rural y rediseñar la institucionalidad para que tenga presencia territorial, así como el mejoramiento del hábitat.

El Objetivo 4: Seguridad, justicia y democracia promueve la seguridad y defensa del territorio nacional; la prestación, la administración y el acceso a los servicios de justicia con un enfoque sistémico y territorial; el fortalecimiento institucional para la promoción, el respeto y la protección de derechos humanos; la construcción de acuerdos sociales incluyentes y la gestión pacífica de conflictos. Estas apuestas se relacionan con los temas de paz, 
TABLA 3. CONVERGENCIA DE LAS ÁREAS DE TRABAJO DE LOS PROYECTOS DE LAS FUNDACIONES AFE CON LOS TEMAS DE LOS OBJETIVOS DEL PLAN NACIONAL DE DESARROLLO 2014-2018

\begin{tabular}{|c|c|c|c|c|c|c|}
\hline & Objetivo 1 & Objetivo 2 & Objetivo 3 & Objetivo 4 & Objetivo 5 & Objetivo 6 \\
\hline $\begin{array}{l}\text { Personas: Educación preescolar, edu- } \\
\text { cación básica y media, Educación supe- } \\
\text { rior y becas, Salud y protección social, } \\
\text { Vida en familia y tejido social, Vivienda } \\
\text { y habitabilidad, Gestión del conoci- } \\
\text { miento, Agua y saneamiento básico } \\
\text { Paz: Cultura y convivencia ciudadana } \\
\text { Prosperidad: Arte y cultura, Recrea- } \\
\text { ción, deporte y uso del tiempo libre, } \\
\text { Promoción de voluntariado, Donacio- } \\
\text { nes y dotación, Inclusión y educación } \\
\text { financiera, Generación de ingresos y } \\
\text { desarrollo productivo, Desarrollo de } \\
\text { base y trabajo con comunidades }\end{array}$ & & 17 & & & & \\
\hline $\begin{array}{l}\text { Paz: Democracia y participación ciuda- } \\
\text { dana, Acceso a la justicia y resolución } \\
\text { pacífica de conflictos, Paz, superación } \\
\text { del conflicto y DD.HH. }\end{array}$ & & & & 4 & & \\
\hline $\begin{array}{l}\text { Personas: Educación técnica, tecno- } \\
\text { lógica y Formación para el trabajo, } \\
\text { Espacios públicos e infraestructura } \\
\text { comunitaria }\end{array}$ & 4 & 3 & & & & \\
\hline $\begin{array}{l}\text { Personas: Seguridad alimentaria y } \\
\text { nutrición }\end{array}$ & & 2 & 2 & & & \\
\hline $\begin{array}{l}\text { Planeta: Gestión ambiental y medio } \\
\text { ambiente, Conservación y desarrollo } \\
\text { sostenible }\end{array}$ & & & & & & 2 \\
\hline $\begin{array}{l}\text { Número total de áreas de trabajo } \\
\text { que convergen con cada objetivo } \\
\text { del PND }\end{array}$ & 6 & 23 & 2 & 5 & 0 & 2 \\
\hline
\end{tabular}

Fuente: elaboración equipo Capstone Universidad Externado de Colombia-AFE 2019, con base en los resultados de la base de proyectos y el PND $2014-2018$. 
democracia, seguridad, justicia, participación e incidencia en políticas públicas, que trabajan las fundaciones AFE.

El Objetivo 5: Buen gobierno, busca fortalecer la articulación nación-territorio; afianzar la lucha contra la corrupción; la transparencia y la rendición de cuentas; promover la eficiencia y la eficacia administrativa y fiscal, además de promover y asegurar los intereses nacionales a través de la política exterior y la cooperación internacional (acuerdos de consulta previa con grupos étnicos). Es de mencionar que no se evidenció ningún área que explícitamente se alinee con lo que plantea este objetivo en el PND. Debido a que este objetivo trata más de la responsabilidad del Estado y de su organización institucional, las fundaciones AFE aún no han identificado cómo aportar al tema social desde este objetivo, por lo cual su portafolio de trabajo no se alinea con este objetivo.

Para el Objetivo 6: Crecimiento verde, que pretende avanzar hacia un crecimiento sostenible y bajo en carbono; sobreproteger y asegurar el uso sostenible ambiental; reducir la vulnerabilidad frente a los riesgos de desastres y el cambio climático, se evidencian dos áreas de trabajo de las fundaciones AFE que se alinean en temas de gestión ambiental y desarrollo sostenible.

Por lo anterior, es evidente la alineación entre las áreas de los proyectos de las fundaciones AfE y los objetivos 1, 2, 3, 4 y 6 del PND 2014-2018, debido a que hay una relación temática directa y explícita. Trabajar alineados con los planes de desarrollo no siempre es fácil y ahí está el verdadero reto, como anotó una fundación empresarial en la entrevista realizada por el equipo Capstone en abril de 2019:

... La labor de los gobiernos es hacer lo que les corresponde en lo concerniente al Plan de Ordenamiento Territorial y políticas públicas, pero se evidencia una desarticulación. Uno de los principales desafíos es trabajar articuladamente, colaborativamente y ser aliados verdaderos, pues muchas veces los actores de los gobiernos llegan desarticulados. (Coordinadora Técnica de una fundación empresarial, comunicación personal, marzo de 2019)

En conclusión, la importancia de reconocer la articulación de las áreas de trabajo con los pilares y objetivos del PND está en visibilizar los aportes del sector fundacional a cada uno, en cuanto a coberturas e inversiones para el cierre de brechas territoriales. Esta información se puede reportar periódicamente al Gobierno de forma consolidada como gremio AFE mediante una metodología estándar para sus procesos de cumplimiento de metas y rendición de cuentas (accountability).

Aquí, la no alineación o desacuerdo en algunos temas aporta a la resolución de problemáticas sociales no priorizadas dentro de las agendas políticas o los ejercicios de planeación territorial, y abre el espacio a procesos de oposición que favorecen la madurez política de la democracia. Asimismo, las organizaciones sociales y los centros de pensamiento pueden cualificar los procesos de ejecución de obras públicas y programas sociales con la autonomía y objetividad de sus análisis, lo que es directa o indirectamente una fuente para el análisis y seguimiento de políticas públicas gubernamentales (Ríos y Garro, 2017). 


\section{Convergencia de las áreas de trabajo de los proyectos de las fundaciones AFE con los ODS}

Para identificar los aportes de las fundaciones AFE al desarrollo sostenible, como se señala en los ODs, se elaboró un análisis de correlación entre los ODS CONPES 3918 (DNP, 2018) y las áreas de trabajo de los proyectos de las fundaciones AFE, así: primero se filtró la matriz de proyectos de las fundaciones AFE por las áreas de trabajo de los proyectos ejecutados entre 2015 y 2018 como lo muestra (figura 3).

De este ejercicio se destacan los siguientes resultados: los cinco primeros temas con más proyectos ejecutados son: 1) vida en familia y tejido social (175 proyectos); 2 ) salud y protección social (151 proyectos); 3) generación de ingresos y desarrollo productivo (145 proyectos); 4) emprendimiento (119 proyectos), y 5) fortalecimiento y entorno institucional (175 proyectos). Lo anterior sin desconocer la importancia del tema educativo, dado que sumados estos cuatro temas: educación básica y media, educación técnica, tecnológica y formación para el trabajo, educación preescolar, y educación superior y becas son 295 proyectos los que apuntan a esta temática.

El segundo paso del ejercicio consistió en tomar cada área de trabajo de los proyectos de las fundaciones AFE y cruzarla con los temas que trata cada uno de los 17 ods, mediante un análisis de relacionamiento textual y cualitativo. Así, cada tema fue evaluado cualitativamente identificando palabras clave y relacionamiento temático con cada oDs. Derivado de ello, se evidenció que todas las áreas de trabajo de los proyectos se alinean de manera explícita o temática con los ods (figura 4).

Al sumar los proyectos que apuntan a cada tema, y teniendo en cuenta los oDs con los cuales están relacionados, se encontró que los cinco primeros oDs con más proyectos relacionados son los objetivos 10: reducción de desigualdades; 11 : ciudades y comunidades sostenibles; 1 : fin de la pobreza; 5 : igualdad de género, y 4: educación de calidad, mientras que los cinco oDs con menos proyectos ejecutados son 9: industria, innovación e infraestructura; 7: energía asequible y no contaminante; 6 : agua limpia y saneamiento básico; 14: vida submarina, y 15: vida de ecosistemas terrestres.

En contraste, los resultados de la encuesta diligenciada por 40 de las 72 fundaciones AFE evidencia un mayor aporte de las fundaciones AFE a nivel de objetivos $(40 \%)$ y temas $(22,5 \%)$, seguido de metas $(20 \%)$ e indicadores $(17,5 \%)$, lo que se podría asociar con la ausencia de una metodología eficiente de seguimiento y evaluación de sus aportes, aun cuando ellas mismas reportan el uso de herramientas de seguimiento, control y reporte de sus actividades y proyectos (figura 5).

$\mathrm{Al}$ corroborar la información de las entrevistas, en efecto, las fundaciones AFE tienen conocimiento de los oDs y se evidencia una disposición por aportar; no obstante, la alineación con los ods no es de manera deliberada en la mayoría de los casos, puesto que las fundaciones AFE operan proyectos derivados de sus líneas de acción y, posteriormente, encuentran por medio de temas los oDs relacionados con sus proyectos, es decir, los oDs se encuentran en la parte inferior de la cadena de ejecución, son el fin mas no el medio. 
FIGURA 3. DISTRIBUCIÓN DE PROYECTOS DE LAS FUNDACIONES AFE POR ÁREAS DE TRABAJO

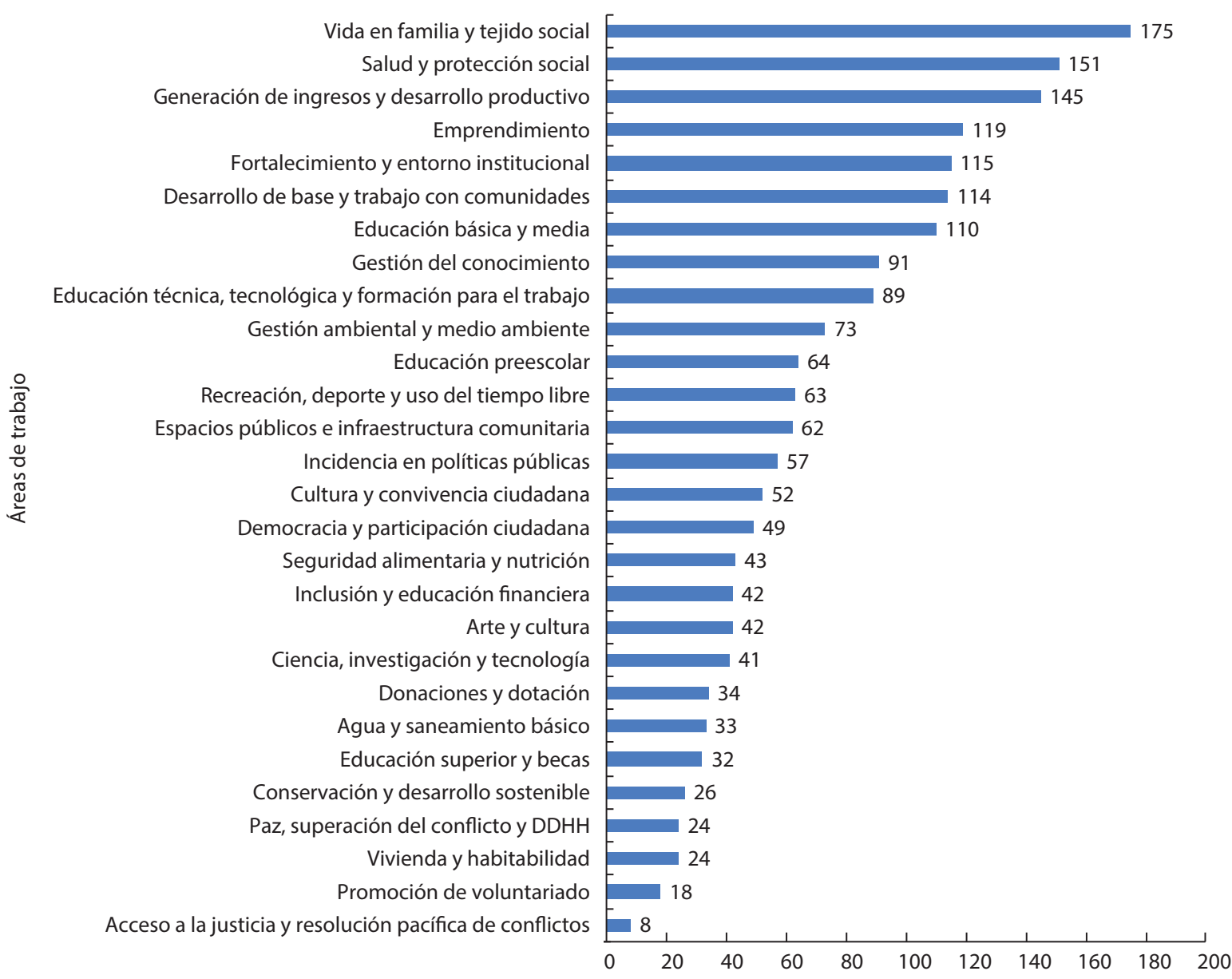

Fuente elaboración equipo Capstone Universidad Externado de Colombia-AFE 2019, con base en los datos de la matriz de proyectos de las fundaciones AFE.

En términos generales, los entrevistados aseguran que el querer lograr la alineación con los 17 oDs y seguir la Agenda 2030 ha dado a la mayoría de las fundaciones la oportunidad de autoevaluarse, de mejorar procesos y de revisar su discurso, porque esto les permite percatarse de que hay una mejor forma de hacer las cosas, ampliando metas y propósitos. Para este tema, la Fundación Promigas y la Fundación Éxito declaran estar aportando a indicadores de los ODS, y otras cuatro fundaciones afirman estar construyendo una metodología para poder reportar. 
FIGURA 4. PUNTUACIÓN DE CADA ODS SUMANDO LAS TEMÁTICAS DE LOS PROYECTOS AFE

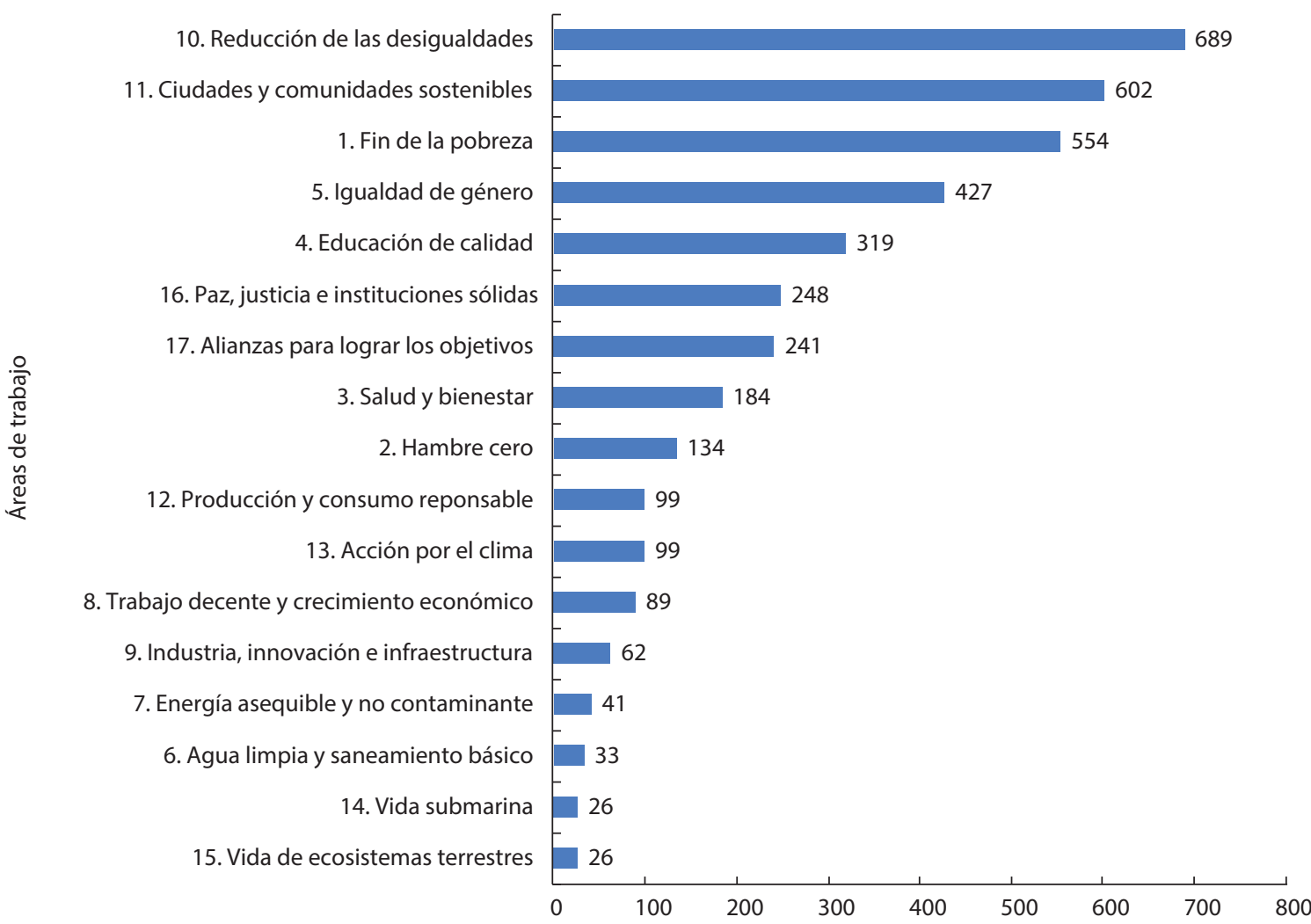

Fuente: elaboración equipo Capstone Universidad Externado de Colombia-AFE 2019, con base en los datos de la matriz de proyectos.

\section{FIGURA 5. NIVEL AL QUE APORTAN LAS FUNDACIONES AFE A LOS ODS}

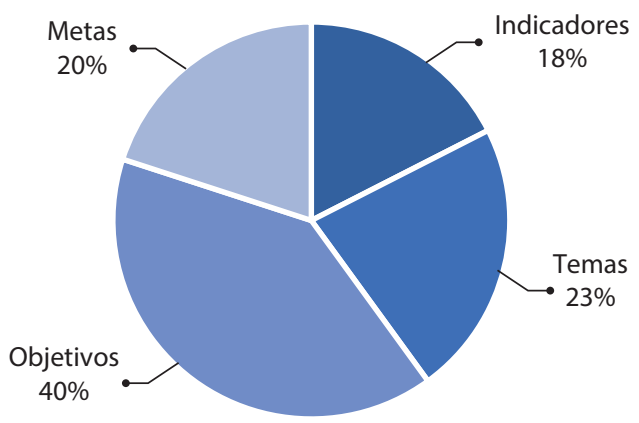

Fuente: elaboración propia con base en resultados de la encuesta Capstone 2018-2019 a fundaciones AFE.

\section{DISCUSIÓN}

Los hallazgos sobre la articulación de las áreas de trabajo de los proyectos de las fundaciones AfE con los ejercicios de planeación del Estado y los oDs aclara el panorama estratégico del sector fundacional. De igual forma, con el análisis de las fuentes de información utilizadas bajo los tres componentes explorados es posible resolver de alguna manera la pregunta ¿cómo se articulan las fundaciones AFE con el desarrollo en los territorios? 
En primer lugar, se puede decir que los proyectos de las fundaciones AFE se articulan en los territorios de acuerdo con criterios de:

1. focalización: sus proyectos de inversión se orientan a sus zonas de influencia, priorizando la ubicación de sus sedes y empresas para el caso de las fundaciones empresariales. En la misma línea, se identificó que una mayor producción de los departamentos (PIB) es un factor que atrae la inversión social de las fundaciones AFE. Un PIB alto no necesariamente implica mayor desarrollo, pero un sistema productivo ineficiente sí puede generar mayores brechas sociales que requieren más inversión social.

Por otro lado, la ubicación geográfica y el acceso a los territorios también es un incentivo para la inversión social; mejor acceso garantiza menos gastos de operación, lo que optimiza la inversión hacia mayores impactos sociales. Por estas circunstancias, el $52 \%$ de los 790 proyectos de las fundaciones AFE se concentra en la zona central del país, principalmente en tres departamentos: Valle del Cauca $(21 \%)$, Antioquia (20\%) y Atlántico (11\%).

2. Alianzas estratégicas: la presencia de actores clave del sector privado o de la cooperación internacional en el territorio favorece el apalancamiento económico, esto es un determinante para la inversión social de las fundaciones AFE en los departamentos, porque a más socios mayores inversiones o colaboraciones, por tanto, más posibilidades de escalonamiento de sus proyectos y mayores impactos sociales. También, una mayor presencia del Estado en los territorios favorece la operación de los proyectos en términos de seguridad, eficiencia, gestión de proyectos y consecución de apoyos o recursos. En este punto es importante mencionar que los departamentos del sur y suroccidente (Meta, Casanare, Arauca, Vichada, Guainía, Guaviare, Caquetá, Vaupés, Putumayo y Amazonas) tienen menor presencia de las fundaciones AFE (menos de 10 proyectos), muy posiblemente porque estos departamentos no cuentan con los criterios mencionados. Actualmente no hay una estrategia puntual sobre esas limitaciones para fomentar la inversión social de todos los sectores.

En segundo lugar, los resultados evidencian una articulación explícita de las áreas de trabajo de los proyectos de las fundaciones AFE con el PND 2014-2018. Esta articulación se da principalmente motivada por beneficios como la voluntad política para ejecutar proyectos en los territorios, la coherencia con los planes de desarrollo de los departamentos, y el cumplimiento de metas sociales de las administraciones locales. Hay una contraprestación para estas administraciones, como es mayor visibilidad de su gestión por el logro de metas comunes, la creación de alianzas estratégicas para la sostenibilidad de sus intervenciones y la generación de capacidad instalada en los territorios para el desarrollo local.

Aquí las áreas de trabajo de las fundaciones AFE que más se alinean con el PND son las que tratan temas relacionados con el desarrollo 
de las personas como: educación, seguridad alimentaria y nutrición, salud y protección social, vida en familia y tejido social, vivienda y habitabilidad, agua y saneamiento básico, y temas que contribuyen a la prosperidad como: espacios públicos e infraestructura comunitaria; ciencia, investigación y tecnología; generación de ingresos y desarrollo productivo; emprendimiento; recreación, deporte y uso del tiempo libre, así como arte y cultura. Los temas que menos se alinean son transformación del campo, crecimiento verde, buen gobierno y paz, temas estructurales que afectan el desarrollo sostenible y son responsabilidad del Estado. Finalmente, como se mencionó, el no estar alineado también puede ser positivo en la medida en que propicia la oposición y el control social; por otro lado, brinda insumos para la rendición de cuentas (accountability), lo que puede cualificar los procesos y ayudar a descubrir nuevas alternativas de intervención para resolver problemas públicos.

En tercer lugar, es evidente que la mayoría de las áreas de trabajo de las fundaciones AFE se alinean temáticamente con los oDs, sobre todo 1, 4 y 5 relacionados con el desarrollo de las personas, y los oDs 10 y 11 asociados a temas de prosperidad; asimismo, se identificó que los ods con menor alineación son 6, 7, 9, 14 y 15 asociados con temas del planeta. A pesar de esto, la articulación en los territorios para temas de ods es desigual. Según el DNP, los departamentos de Atlántico, Bolívar, Cesar, Sucre, Córdoba, La Guajira, Magdalena, Chocó, Nariño y Cauca requieren mayores esfuerzos para el logro de los ods 1, 2, 3, 4, 5 asociados al desarrollo de las personas; el oDs
6 asociado al desarrollo del planeta, y los oDs 9, 10, 11 asociados a la prosperidad (cálculos del DNP basados en las metas que marca el CONPES 3918 de 2018). En contraposición a estas cifras, las fundaciones AFE cobijan departamentos como Cesar, Sucre y Chocó con menos de 20 proyectos, y en los departamentos de Meta, Casanare, Arauca, Vichada, Guainía, Guaviare, Caquetá, Vaupés, Putumayo y Amazonas las fundaciones AFE hacen presencia con menos de 10 proyectos. En este punto se debe tener en cuenta que para estas zonas las metas del CONPES 3918 son más aterrizadas, sin embargo, hay rezagos importantes para el logro de los ods 3, 4, 7, 16 en 2030 y sería deseable mayor cobertura de la inversión social en estos territorios para temas de salud, bienestar, educación, paz y energías sostenibles.

Con todo lo anterior, se confirma la hipótesis planteada en relación con la no coherencia entre la focalización de los proyectos de las fundaciones AFE y la pertinencia temática de sus áreas de trabajo con el PND 2014-2018 para el cumplimiento de las metas de los ods, por lo cual un direccionamiento más estratégico traería mayores impactos sociales que contribuyan al desarrollo en los territorios.

\section{CONCLUSIONES}

Este artículo ilustra sobre la focalización de la inversión social privada de las fundaciones AFE a nivel de proyectos por departamento, así como los temas priorizados por las fundaciones armonizados con el PND 2014-2018 y su articulación con los 17 oDs, lo que permite 
identificar los territorios menos intervenidos y resalta las necesidades temáticas reflejadas en mayores rezagos frente al cumplimiento de metas del conPes 3918. Los datos presentados aquí visibilizan las brechas frente a las metas de oDs en 2030 para cada departamento, y el grado de esfuerzo que requieren expresado en puntos porcentuales. Esto permite cualificar los procesos de focalización de las áreas de trabajo que desarrollan los proyectos en los departamentos.

Por otro lado, las respuestas a los cuestionarios aplicados en las entrevistas exponen los criterios que las fundaciones usan para priorizar los territorios en donde implementarán sus proyectos y las alianzas deseables para que estos obtengan mejores resultados e impactos. En este orden, el cruce de las áreas de trabajo de los proyectos de las fundaciones AFE con los tres pilares y los seis objetivos del PND 2014-2018 deja ver los temas que no han sido cubiertos y que son prioridad para el Estado en ese periodo. Aquí es clave revisar si estos temas desatendidos han sido priorizados en el PND 2018-2022 del nuevo gobierno, y si es pertinente su inclusión en los proyectos por ejecutar en este nuevo periodo. Queda abierta la inquietud de cómo trabajar los reportes de aportes a metas o indicadores locales y nacionales para los planes de desarrollo y los oDs dentro de las alianzas entre las fundaciones e instituciones del Estado.

\section{REFERENCIAS}

Banco Interamericano de Desarrollo (BID) (2018). Informe de sostenibilidad 2018. Recuperado de https:// publications.iadb.org/es/banco-interamericanode-desarrollo-informe-de-sostenibilidad-2018

Carvajalino, G. (2007). Inversión social privada en América Latina: retos y desafíos. Ensayo presentado en la Jornada sobre Inversión Social Privada de la Fundación Minetti. Bogotá: Gestratégica. Recuperado de http://www.gestrategica.org/ templates/listado_recursos.php?id_rec=28\&id

Chávez, D. y Yepes, G. (2012). Principios de inversión social. Experiencias de los participantes del Pacto Mundial en América Latina y el Caribe. Bogotá: Centro Regional para América Latina y el Caribe. Recuperado de https://administracion.uexternado.edu.co/matdi/Otros/responsabilidadSocial/ herramientas/8.\%20RsE\%20Casos\%20IsBN.pdf

Departamento Nacional de Planeación (DNP) (2014). Bases del PND 2014-2018. Recuperado de https:// colaboracion.dnp.gov.co/CDT/Prensa/Bases $\% 20$ Plan\%20Nacional\%20de\%20Desarrollo\%20 2014-2018.pdf

Departamento Nacional de Planeación (DNP) (2017). La Agenda 2030 en Colombia (Gubernamental). Bogotá: DNP.

Departamento Nacional de Planeación (DNP) (2018). CONPES 3918 Estrategia para la implementación de los (ODS) en Colombia. Recuperado de https://colaboracion.dnp.gov.co/CDT/CONPES/ Econ\%C3\%B3micos/3918.pdf 
Departamento Nacional de Planeación (DNP) (2019). La agenda 2030 en Colombia. Recuperado de https://www.ods.gov.co/es/objetivos/fin-dela-pobreza

Flores, M. (2007). La identidad cultural del territorio como base de una estrategia de desarrollo sostenible. Revista Opera, (7), 35-54.

Fundación Promigas y la Fundación DIs (2012). Las fundaciones empresariales en Colombia: una aproximación a su estructura y dinámicas. Recuperado de http://grupodis.net/docs_gestrategica/ Presentaci\%C3\%B2n\%20Fundaciones\%20 Empresariales\%20en\%20Colombia_Fund_ DIs_2012.pdf

Gazmuri, C. (2002). Taller: accountability de las organizaciones de la sociedad civil. Chile. Recuperado de http://www.prohumana.cl/minisitios/feria/ presenta/cgazmuri2.pdf

Global Impact Investment Network (GIIN) (2017). What you need to know about impact investing. Recuperado de https://thegiin.org/impact-investing/ need-to-know/

Haro-Martínez, A. A. y Taddei-Bringas, I. C. (2014). Sustentabilidad y economía: la controversia de la valoración ambiental. Economía, sociedad $y$ territorio, 14(46), 743-767. Recuperado de http://www.scielo.org.mx/scielo.php?script=sci_ arttext\&pid=S1405-84212014000300007\&ln $\mathrm{g}=\mathrm{es} \& \mathrm{t} \operatorname{lng}=\mathrm{es}$.

Impacto, I. d. (2017). ¿Inversión de impacto en $A r-$ gentina 2017: oportunidades y desafios. Buenos Aires: Fomin.

Isunza Vera, E. (2006). Para analizar los procesos de democratización: interfaces socioestatales, proyectos políticos y rendición de cuentas. En E. Isunza Vera y A. J. Olvera (coords.). Democratización, rendición de cuentas y sociedad civil: participación ciudadana y control social. México:
CIESAS/Universidad Veracruzana/Miguel Ángel Porrúdea/Cámara de Diputados, LIx Legislatura (colección Conocer para Decidir).

Natal, A. (2006). Rendición de cuentas: apuntes para una revisión conceptual. Documentos de discusión sobre el nuevo institucionalismo. Ciudad de México: El Colegio Mexiquense.

Ordóñez, G. (2013). Manual de análisis y diseño de políticas públicas. Bogotá: Universidad Externado de Colombia.

Organización de las Naciones Unidas para la Alimentación y la Agricultura (faO) (2013). Enfoque territorial participativo, metodología válida para el diseño de emprendimientos estratégicos articuladores vinculados a la Soberanía y Seguridad Alimentaria. Recuperado de http://www.fao. org/3/a-as831s.pdf

Organisation for Economic Co-operation and Development (OECD) (2015). Social Impact Investment. Building the Evidence Base. OECD Publishing, Paris, https://doi.org/10.1787/9789264233430-en

Organización para la Cooperación y el Desarrollo Económicos (OECD) (2016). Colaboración entre fundaciones y el gobierno: Evidencias desde México. París: Centro de Desarrollo de la OECD. Recuperado de https://www.oecd.org/dev/developmentphilanthropy/netFwD-Mexico-es-ebook.pdf

Programa de las Naciones Unidas para el Desarrollo (PNUD) (2018). ODS en Colombia: retos para 2030. Recuperado de https://www.undp.org/ content/dam/colombia/docs/ods/undp_co_ PUBL_julio_ODs_en_Colombia_los_retos_para_2030_onU.pdf

Programa de las Naciones Unidas para el Desarrollo (pNud) (2019). Antecedentes. Recuperado de https://www.undp.org/content/undp/es/home/ sustainable-development-goals/background. html 
Ríos Ramírez, A. y Garro, J. E. (2017). Accountability y sociedad civil: el control político en la era digital. Papel Político, 22(2), 311-337. Recuperado de http://www.scielo.org.co/pdf/papel/ v22n2/0122-4409-papel-22-02-00311.pdf

Sinigaglia, et al. (2006). Actuar para cambiar: estrategias de incidencia de las organizaciones sociales en las politicas públicas. Recuperado de https:// es.slideshare.net/FARCOARG/actuar-para-cambiar

Villar Gómez, R. (2018). Las fundaciones en Colombia. Características, tendencias, desafíos. Asociación de Fundaciones Familiares y Empresariales - AFE Colombia. Bogotá: Gente Nueva.
Villar Gómez, R. (2015). Recursos privados para la transformación social. Filantropía e inversión social privada en América Latina hoy. Argentina, Brasil, Colombia y México. Argentina: AfE y Grupo de Fundaciones y Empresas (GDFE); Brasil: Grupo de Institutos, Fundaciones y Empresas (GIFE); Colombia: Afe de Colombia; México: Centro Mexicano para la Filantropía (Cemefi). 